\title{
Does human P-glycoprotein efflux involve transmembrane alpha helix breakage?
}

\author{
Authors: Cátia A. Bonito ${ }^{1,2}$, Maria-José U. Ferreira ${ }^{2}$, Ricardo J. Ferreira ${ }^{2,3}$,* Daniel J. V. A. dos \\ Santos ${ }^{1,2,4, *}$
}

Affiliations:

${ }^{1}$ LAQV@REQUIMTE, Department of Chemistry and Biochemistry, Faculty of Sciences, University of Porto, Rua do Campo Alegre, 4169-007, Porto, Portugal.

${ }^{2}$ Research Institute for Medicines (iMed.ULisboa), Faculty of Pharmacy, Universidade de Lisboa, Avenida Prof. Gama Pinto, 1649-003 Lisboa, Portugal.

${ }^{3}$ Department of Cell and Molecular Biology - Molecular Biophysics, Biomedicinskt centrum (BMC), Uppsala University, Husargatan 3, 75237 Uppsala, Sweden.

${ }^{4}$ BioISI: Biosystems and Integrative Sciences Institute, Faculty of Sciences, University of Lisbon, Campo Grande, C8, 1749-016, Lisbon, Portugal.

\section{*Correspondence to: ricardo.ferreira@icm.uu.se or ddsantos@fc.up.pt}

Abstract: The occluded conformation suggested in a recent article that revealed a new inwardfacing conformation for the human P-glycoprotein may not represent the closing of a gate region but instead an artifact derived from lateral compression in a too small sized nanodisc, used to stabilize the transmembrane domains of the transporter.

A research article published by Locher and co-workers [1] turns an important page in studying $\mathrm{ABC}$ transporters because it is the first cryo-EM inward-facing structure of human Pglycoprotein (P-gp), obtained with both substrate and inhibitor bound, that together with the outward-facing ATP-bound human P-gp published in 2018 [2] allow a more complete overview on the conformational changes associated to the efflux cycle. However, the claiming by the authors that authors state that "... an occluded conformation (...) in a central cavity formed by closing of a gate region consisting on TM4 and TM10" must be thoroughly discussed. Although correct from the commonly accepted mechanistic point of view, the presence of such discontinuities in both helices is strikingly different from all other published structures [3-8], in which both TM4 and/or TM10 are depicted as full helical domains. As under normal bending forces the breakage of 
hydrogen bonds that hold the helical structure together should not occur [9], it is important to try clarifying the underlying reason.

This specific feature had already been reported for a murine P-gp structure [8] in the presence of cyclopeptides from the QZ series. Together with ATPase and Calcein-AM transport assays, Szewczyk and co-workers proposed that the structural kink observed in TM4 (but not TM10) were induced by ligands that function more as activators of ATPase (QZ-Ala and QZ-Val) while non-activators (QZ-Leu and QZ-Phe) failed to induce the TM4 kink. However, a previous P-gp structure also co-crystallized with QZ-Val failed to show any kink on either TM4 or TM10 $[3,10]$. This way, the comparison between both murine and human structures suggests three additional questions: 1) if related to ligand entry, why did not TM10 kinked as TM4 after the passage of the QZ activators through the TM4/TM6 portal in the murine structure?, 2) by which gate taxol entered into hP-gp, because both TM4 and TM10 are kinked and 3) if related to ATPase activators, why did zosuquidar (inhibitor, $\mathrm{IC}_{50} 60 \mathrm{~nm}$ ) [11] induced a similar TM4/TM10 kink as substrates do? Most intriguingly, the proposed occluded conformation also impairs the regulation of the inherent substrate specificity of P-gp by a small linker region (missing in all structures) $[12,13]$ because it becomes unable to directly interact with substrates.

To answer all these questions, we started by comparing all P-gp (ABCB1) structures available in the Protein Data Bank (Table 1). It is possible to verify that, while NBD-NBD distances in murine P-gp structures are within $48 \AA$ (4M1M) up to $65 \AA$ (4Q9K), in the inwardfacing human P-gp, this distance reduces to only $34 \AA$ (bound to zosuquidar) or $37 \AA$ (bound to taxol), only 7-8 $\AA$ greater than those registered for the outward-facing P-gp structures reported to date $(28 \AA)$ [2,14]. However, as these structures were obtained without the presence of ATP, how can we explain such reduction in NBD-NBD distances? More interestingly, another murine P-gp structure also obtained by cryo-EM reported a distance of $55 \AA$, well within the ones reported previously in the other crystallographic structures. When reporting only to the cryo-EM structures, both inward murine (PDB ID: 6GDI) and outward human (PDB ID: 6C0V) were found to have both TM4/TM10 modelled as straight helices. Furthermore, while Thongin and co-workers identified electron densities compatible with "detergent head-groups from the annular detergent micelle (...) close to two regions predicted to delineate two pseudo-symmetry-related drug-binding sites" but no TM4/TM10 kink [5], other authors reported for the outward-facing human structure 
that the "continuity of these helices is important to completely close the intracellular gate upon NBD dimerization, avoiding potential leakage in the outward-facing state" [2]. So, what could be the underlying reason for such a difference?

Table 1. Comparison of P-glycoprotein structures available in the Protein Data Bank.

\begin{tabular}{|c|c|c|c|c|c|c|c|}
\hline PDB ID & Organism & Ligand & Class & Conformation & $\begin{array}{c}\text { NBD- } \\
\text { NBD (A) }\end{array}$ & $\begin{array}{c}\text { Distorted } \\
\text { TM's }\end{array}$ & Type \\
\hline $3 \mathrm{WME}$ & C. merolae & -- & -- & inward & 42.59 & -- & crystal \\
\hline $3 \mathrm{WMG}$ & C. merolae & $\mathrm{aCAP}$ & -- & inward & 42.59 & 4 & crystal \\
\hline $4 \mathrm{~F} 4 \mathrm{C}$ & C. elegans & $\begin{array}{c}\text { undecyl b-D- } \\
\text { thiomaltopyranoside }\end{array}$ & detergent & inward & 58.82 & $3,9,10,12$ & crystal \\
\hline $4 \mathrm{M} 1 \mathrm{M}$ & M. musculus & -- & -- & inward & 48.03 & 12 & crystal \\
\hline $4 \mathrm{M} 2 \mathrm{~S}$ & M. musculus & QZ59-Val (RRR) & inhibitor & inward & 48.32 & 12 & crystal \\
\hline 4Q9H & M. musculus & -- & -- & inward & 61.85 & -- & crystal \\
\hline 4Q9I & M. musculus & QZ-Ala (x2) & substrate & inward & 59.80 & 4 & crystal \\
\hline 4Q9J & M. musculus & QZ-Val (x2) & substrate & inward & 62.56 & 4 & crystal \\
\hline $4 Q 9 K$ & M. musculus & QZ-Leu & inhibitor & inward & 65.38 & -- & crystal \\
\hline 4Q9L & M. musculus & QZ-Phe & inhibitor & inward & 60.00 & -- & crystal \\
\hline 4XWK & M. musculus & BDE-100 & pollutant & inward & 60.69 & -- & crystal \\
\hline $5 \mathrm{KPI}$ & M. musculus & -- & -- & inward & 47.99 & 12 & crystal \\
\hline $6 \mathrm{~A} 6 \mathrm{M}$ & C. merolae & -- & -- & outward & 27.65 & -- & crystal \\
\hline $6 \mathrm{~A} 6 \mathrm{~N}$ & C. merolae & -- & -- & inward & 43.19 & 4 & crystal \\
\hline $6 \mathrm{COV}$ & H. sapiens & ATP & nucleotide & outward & 28.28 & -- & cryo-EM \\
\hline 6FN1 & chimera & zosuquidar (x2) & inhibitor & inward & 34.14 & $4,10,12$ & cryo-EM * \\
\hline 6QEX & H. sapiens & taxol & substrate & inward & 36.72 & $4,10,12$ & cryo-EM * \\
\hline 6GDI & M. musculus & -- & -- & inward & 55.19 & -- & cryo-EM \\
\hline
\end{tabular}

* Obtained inserted in a lipid nanodisc.

Quite remarkably, the human P-gp structures reported by Locher and co-workers are the first ones in which a nanodisc was employed to maintain a hydrophobic environment around the transmembrane helices, while in all others detergent micelles were used. Nanodiscs are derived from apolipoprotein A1, also called membrane scaffolding protein (MSP), an amphipathic protein in which the hydrophobic helices interact with the acyl chains of the lipids while the hydrophilic sides are exposed to the solvent, thus allowing the formation of a lipid bilayer patch in solution. However, it was recently reported that the nanodisc lipid internal dynamics and thermotropism may be substantially altered, abolishing gel-fluid phase transitions and increasing site-specific order parameters that can only be fine-tuned with the addition of cholesterol [15]. As it is known that P-gp reshapes the surrounding lipid environment up to a radius of 15-20 nm [16], and as the inferred nanodisc size is $\sim 10 \mathrm{~nm}$ (containing 120-160 lipids) [15], under the temperature conditions required by cryo-EM and using relatively small nanodiscs, the protein conformational space may be perturbed. 
Although other papers already reported murine [17,18] and human [19] P-gp activities reconstituted in nanodiscs, we identified at least two main differences in nanodisc preparation.

To provide a proof of concept, we have generated a series of molecular dynamics (MD) simulations to mimic the effect of lateral compression by a nanodisc under low-temperature conditions. To speed up the calculations all waters were removed, phosphate atoms were spatially restrained in $z$ (xy corresponds to the membrane plane) to prevent membrane disassembling and a lateral pressure of 1.5 bar ( $x y$ only) was applied, using an anisotropic pressure coupling scheme. To further promote lipid order parameters, we additionally decreased the membrane temperature $(280 \mathrm{~K})$ while keeping the protein at $310 \mathrm{~K}$. Two systems were simulated, in the absence or presence of a ligand. From Figure 1, it is possible to verify that, in the absence of ligands, only one helix was found to bend, in this case TM10 (Figure 1A), similar to what was found for the murine 
structures published by Szewczyk and co-workers. When in the presence of a large ligand as cyclosporin (with a MW above $600 \mathrm{Da}$, as taxol), both TM4 (Figure 1B) and TM10 (Figure 1C) helices were found to bend inwards, immediately below the ligand and apparently 'closing' the access to additional molecules. This is clear from the decrease in the root-mean square deviation between both helices regarding the human P-gp cryo-EM structure and the human P-gp homology model, shifting from $4.61 \AA$ to $3.24 \AA$ in TM10 and from $4.21 \AA$ to $2.89 \AA$ in TM4. The distance between TM4 and TM10 was also found to decrease during the 100 ns run, from $18.8 \AA$ to 13.4 $\AA$. Thus, our simulations suggest that by applying a mild increment on the lateral pressure, TM4 and TM10 tend to bend inwards immediately below the substrate, as observed in the cryo-EM structure by Locher and co-workers.
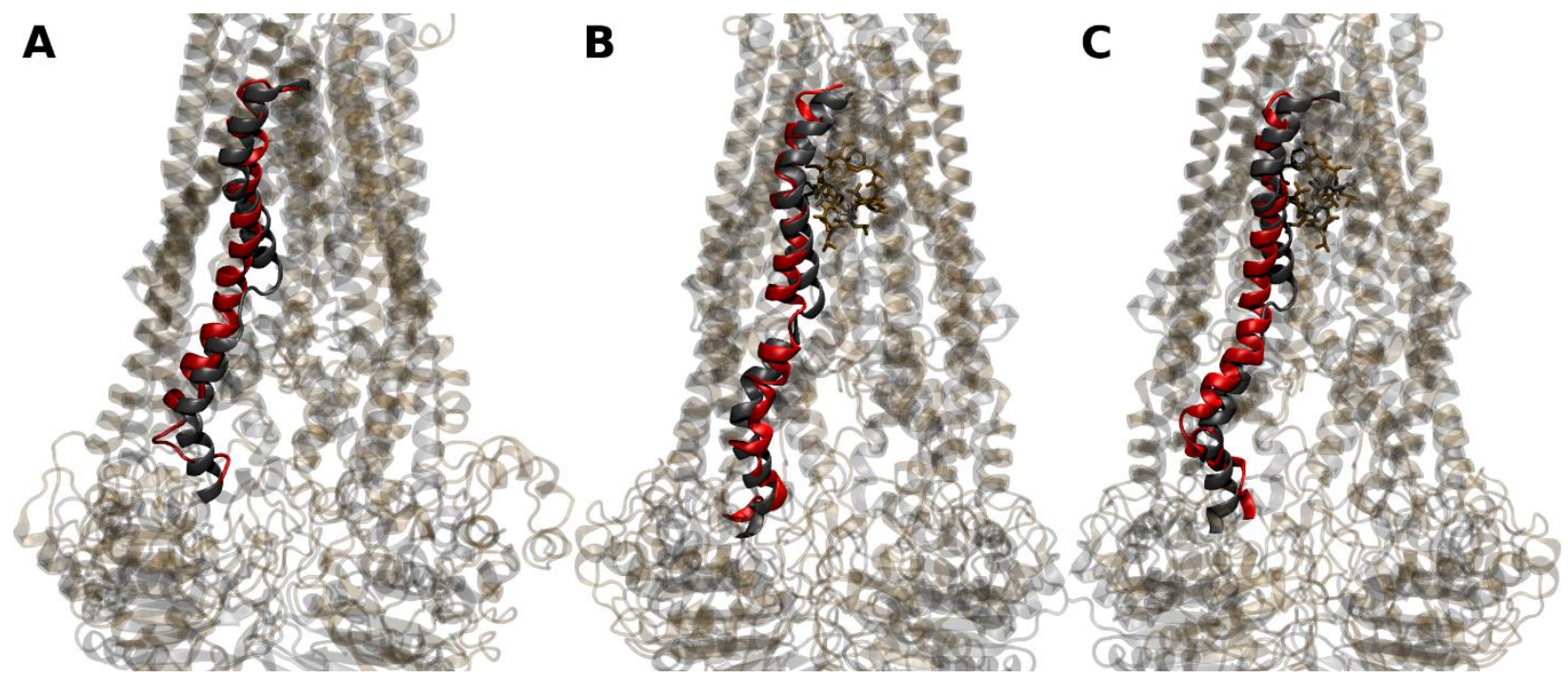

Figure 1. Superimposition of human P-gp (PDB ID: 6QEX, grey) with the final conformations of molecular dynamics runs starting with a homology P-gp model (red). (A), apo structures (TM10 depicted); holo structures depicting taxol (grey) and cyclosporine (ochre) together with TM4 (B) or TM10 (C).

Interestingly, in the murine structures previously reported only when two QZ molecules were found at the drug-binding site (combined MW, $1212 \mathrm{Da}$ ) a TM4 kink was present, and in our simulations only in the presence of bound molecules such kinks were formed. More surprisingly, it was only necessary to apply a slightly higher pressure (1.1 bar) to initiate such changes, becoming more apparent when using 1.5 bar. Furthermore, we additionally observed that this effect seems to occur quite fast in the time scale of the simulations, as we were able to obtain similar results using short and harsher simulation conditions (1 ns each) or longer simulations (100 ns), using more mild conditions ( 0.2 bar increments over $0.5 \mathrm{~ns}$ until reaching $1.5 \mathrm{bar}$ ). In other 
words, even when the pressure increment is slower and occurs over a longer time, the distortion on TM4 and 10 occurs promptly when in the presence of a large ligand at the internal drug-binding pocket.

Therefore, it is our opinion that, and unlike the initially postulated hypothesis, the observed 'gate closing' in the recently published cryo-EM human P-glycoprotein structure may be an artifact from a too small nanodisc construct used for stabilizing the transmembrane domains of the transporter. Under certain cryo-EM conditions, the internal lipid dynamics provided by the nanodisc may render unfavorable conditions for the structural stability of the transporters' architecture, leading to severe distortions of TM4 and TM10 helices. This also upholds the importance of a careful choice of the experimental conditions and protocol to be used to obtain reliable models of membrane proteins since these cryo-EM structures are establishing new and improved starting points for improved computer simulations [21-23].

\section{Acknowledgement}

Fundação para a Ciência e Tecnologia (FCT) is acknowledged for financial support (PTDC/MED-QUI/30591/2017, PTDC/MED-QUI/28800/2017 and SAICTPAC/0019/2015). Cátia A. Bonito also acknowledges FCT for the Ph.D grant SFRH/BD/130750/2017.

\section{References:}

1 Alam A, Kowal J, Broude E, Roninson I \& Locher KP (2019) Structural insight into substrate and inhibitor discrimination by human P-glycoprotein. Science 363, 753-756.

2 Kim Y \& Chen J (2018) Molecular structure of human P-glycoprotein in the ATP-bound, outward-facing conformation. Science 359, 915-919.

3 Li J, Jaimes KF \& Aller SG (2014) Refined structures of mouse P-glycoprotein. Protein Sci 23, 34-46.

4 Ward AB, Szewczyk P, Grimard V, Lee C-W, Martinez L, Doshi R, Caya A, Villaluz M, Pardon E, Cregger C, Swartz DJ, Falson PG, Urbatsch IL, Govaerts C, Steyaert J \& Chang G (2013) Structures of P-glycoprotein reveal its conformational flexibility and an epitope on the nucleotide-binding domain. Proc Natl Acad Sci USA 110, 13386-91.

5 Thongin N, Collins RF, Barbieri A, Shafi T, Siebert A, Ford RC, Thonghin N, Collins RF, Barbieri A, Shafi T, Siebert A \& Ford RC (2018) Novel features in the structure of Pglycoprotein $(\mathrm{ABCB} 1)$ in the post-hydrolytic state as determined at $7.9 \AA$ resolution. $B M C$ Struct Biol 18, 17. 
6 Nicklisch SCT, Rees SD, McGrath AP, Go kirmak T, Bonito LT, Vermeer LM, Cregger C, Loewen G, Sandin S, Chang G \& Hamdoun A (2016) Global marine pollutants inhibit Pglycoprotein: Environmental levels, inhibitory effects, and cocrystal structure. Sci Adv 2, e1600001-e1600001.

7 Esser L, Zhou F, Pluchino KM, Shiloach J, Ma J, Tang W-K, Gutierrez C, Zhang A, Shukla S, Madigan JP, Zhou T, Kwong PD, Ambudkar S V., Gottesman MM \& Xia D (2017) Structures of the Multidrug Transporter P-glycoprotein Reveal Asymmetric ATP Binding and the Mechanism of Polyspecificity. J Biol Chem 292, 446-461.

8 Szewczyk P, Tao H, McGrath AP, Villaluz M, Rees SD, Lee SC, Doshi R, Urbatsch IL, Zhang Q \& Chang G (2015) Snapshots of ligand entry, malleable binding and induced helical movement in P-glycoprotein. Acta Crystallogr D Biol Crystallogr 71, 732-741.

9 Choe S \& Sun SX (2005) The elasticity of $\alpha$-helices. J Chem Phys 122, 244912.

10 Aller SG, Yu J, Ward A, Weng Y, Chittaboina S, Zhuo R, Harrell PM, Trinh YT, Zhang Q, Urbatsch IL \& Chang G (2009) Structure of P-glycoprotein reveals a molecular basis for polyspecific drug binding. Science 323, 1718-1722.

11 Dantzig AH, Shepard RL, Cao J, Law KL, Ehlhardt WJ, Baughman TM, Bumol TF \& Starling JJ (1996) Reversal of P-glycoprotein-mediated multidrug resistance by a potent cyclopropyldibenzosuberane modulator, LY335979. Cancer Res 56, 4171-9.

12 Sato T, Kodan A, Kimura Y, Ueda K, Nakatsu T \& Kato H (2009) Functional role of the linker region in purified human P-glycoprotein. FEBS J 276, 3504-16.

13 Ferreira RJ, Ferreira M-JU \& dos Santos DJVA (2013) Assessing the Stabilization of PGlycoprotein's Nucleotide-Binding Domains by the Linker, Using Molecular Dynamics. Mol Inf 32, 529-540.

14 Kodan A, Yamaguchi T, Nakatsu T, Matsuoka K, Kimura Y, Ueda K \& Kato H (2019) Inwardand outward-facing X-ray crystal structures of homodimeric P-glycoprotein CmABCB1. Nat Comms 10, 88.

15 Martinez D, Decossas M, Kowal J, Frey L, Stahlberg H, Dufourc EJ, Riek R, Habenstein B, Bibow S \& Loquet A (2017) Lipid Internal Dynamics Probed in Nanodiscs. ChemPhysChem 18, 2651-2657.

16 Ferreira RJ, dos Santos DJVA \& Ferreira M-JU (2015) P-glycoprotein and membrane roles in multidrug resistance. Future Med Chem 7, 929-946.

17 Li MJ, Nath A \& Atkins WM (2017) Differential Coupling of Binding, ATP Hydrolysis, and Transport of Fluorescent Probes with P-Glycoprotein in Lipid Nanodiscs. Biochemistry 56, 2506-2517.

18 Li MJ, Guttman M \& Atkins WM (2018) Conformational dynamics of P-glycoprotein in lipid nanodiscs and detergent micelles reveal complex motions on a wide time scale. J Biol Chem 293, 6297-6307.

19 Ritchie TK, Kwon H \& Atkins WM (2011) Conformational Analysis of Human ATP-binding Cassette Transporter ABCB1 in Lipid Nanodiscs and Inhibition by the Antibodies MRK16 
and UIC2. J Biol Chem 286, 39489-39496.

20 Dastvan R, Mishra S, Peskova YB, Nakamoto RK \& Mchaourab HS (2019) Mechanism of allosteric modulation of P-glycoprotein by transport substrates and inhibitors. Science $\mathbf{3 6 4}$, 689-692.

21 Ferreira RJ, Ferreira M-JU \& dos Santos DJVA (2012) Insights on P-Glycoprotein's Efflux Mechanism Obtained by Molecular Dynamics Simulations. J Chem Theory Comput 8, 18531864.

22 Ferreira RJ, Bonito CA, Ferreira MJU \& dos Santos DJVA (2017) About P-glycoprotein: a new drugable domain is emerging from structural data. WIREs Comput Mol Sci 7, e1316.

23 Ferreira RJ, Bonito CA, Cordeiro MNDS, Ferreira M-JU \& dos Santos DJVA (2017) Structurefunction relationships in ABCG2: insights from molecular dynamics simulations and molecular docking studies. Sci Rep 7, 15534.

Author contributions: R. J. F. and D. J. V. A. S. formulated the hypothesis. C.A.B., R. J. F. and D.J.V.A.S. written the manuscript. R. J. F. performed the MD simulations and rendered the images. All authors revised the manuscript and agree with its final form.

Competing interests: All authors declare no competing interests. 

$\left.80^{2}\right)^{2}$

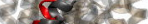
$5.5 \times 2: 9$ \&. 20 1 s 19 Sh 19 3 - Sustoris. ney 2896

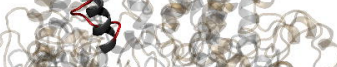
$(8)$ $35 x^{2}=$ s. $52: 538$ sosters $x+52 x$

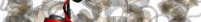
cos 200 55 . seres $6023-4 \times$ (6)

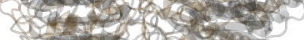

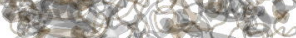

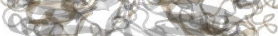

C

$-2020$

$5+25$ $34 x+3$ - 6.925

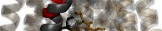
$3.59+215$ 1. H. $\rightarrow+40$.

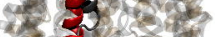
$42 \pi+25$ a $y>$ and an

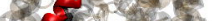
is $27+9$

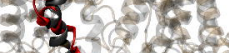

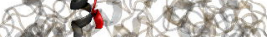

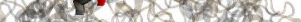

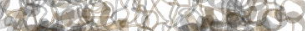

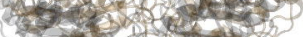

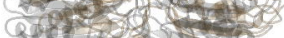

